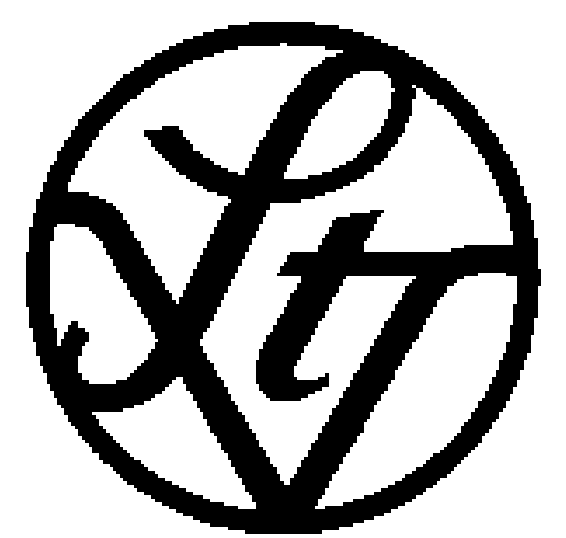

J. A. P. Schulz' "daenifche" Oper

Author(s): Max Seiffert and J. A. P. Schulz

Source: Archiv für Musikwissenschaft, 1. Jahrg., H. 3. (Apr., 1919), pp. 422-431

Published by: Franz Steiner Verlag

Stable URL: http://www.jstor.org/stable/929977

Accessed: 16/06/2014 01:29

Your use of the JSTOR archive indicates your acceptance of the Terms \& Conditions of Use, available at http://www.jstor.org/page/info/about/policies/terms.jsp

JSTOR is a not-for-profit service that helps scholars, researchers, and students discover, use, and build upon a wide range of content in a trusted digital archive. We use information technology and tools to increase productivity and facilitate new forms of scholarship. For more information about JSTOR, please contact support@ jstor.org.

Franz Steiner Verlag is collaborating with JSTOR to digitize, preserve and extend access to Archiv für Musikwissenschaft. 


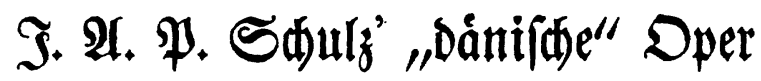

\author{
Boll \\ Mar Seiffert, Berlin
}

\begin{abstract}
$\mathfrak{I}$

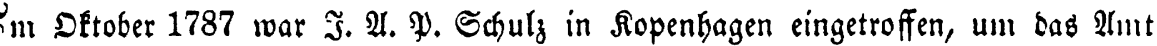

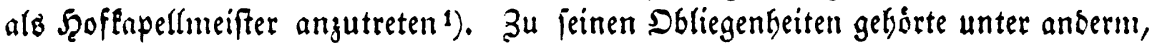

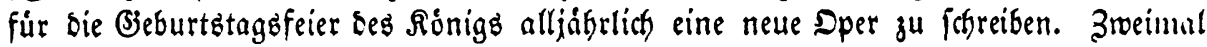

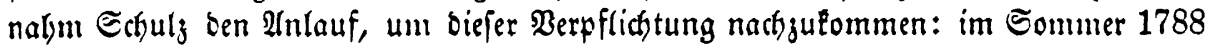
erwartete er zu siejem Zwecte ein Tertbuct) bes Dichters Thomas Tharap, uns

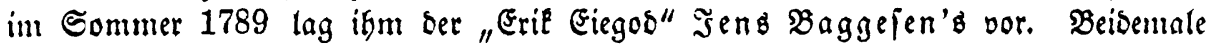
gelangte jebod) Edyul nicht zum Biel. Mit bem Tertbuch Baggefen's nus Brrủnben,

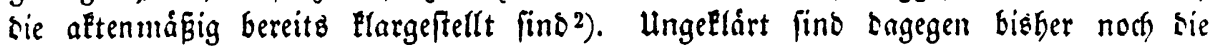

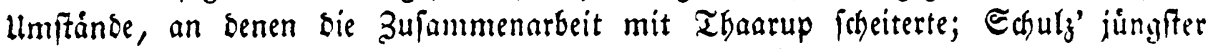
Biograph geht jebenfalls mit zuei belanglojen zeilen úber fie hinweg, augenlfheinlich,

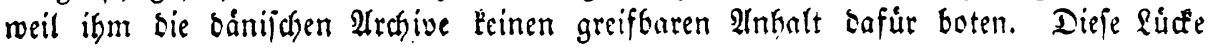
foll in golgenden ausgefúllt werben.

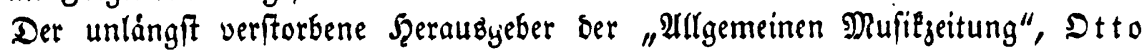

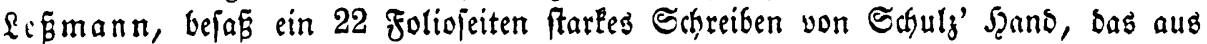

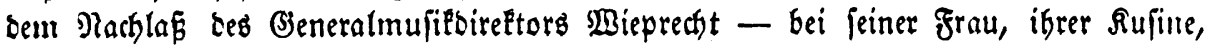
verlebte Echulz" Zochter "Winchen" ifren Rebensabeno - in feinen Befizge gelangt

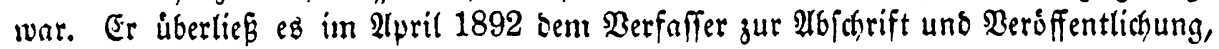
obne sás siejer B̈elegenteit gefunden bảtte, bavon geeigneten biebrauch zu machen.

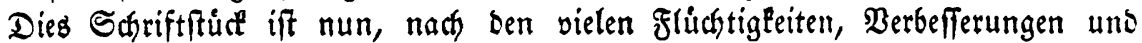
Piandeinjchaltungen ju urteilen, offenbar ber Entwurf zu einem Rechtfertigungs= ichreiben geweien, mit sem Safulz gerwiffen 2fnidulsigungen Thararup's entgegentrut uno beffen Meinjichrift wohl ber groß̈e Brand Ropenhagens vernidjtete. Sein sngalt

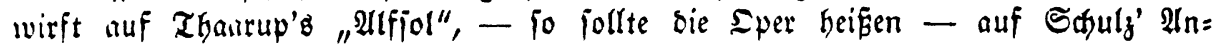
ichauungen úber Dpernfompofition uns feinen menichlichen Sbarafter ein io begeids=

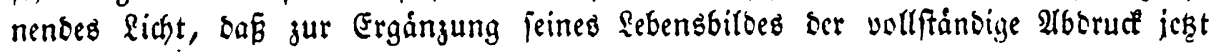
ausericheno begrúndet ericheint.
\end{abstract}

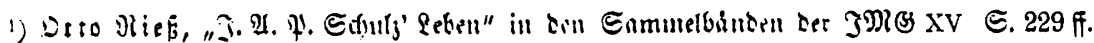

2) ล. ก. D. S. 235 开. 


\section{-Pro Memoria.}

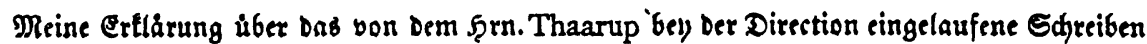
ift leid)t gemadt. Jd Darf nur erjảglen, was vorgegangen, und was ber Direstion fhon jum Egeil betannt ift.

Im Dejember bes $1787^{\mathfrak{n}}$ Jahres engagirte fid Şr. Th. in Sr. Excellenj bes Şerrn Dber: bofmaridjall Numsen und meiner Begenwart sine Dper ju madjen, bie idj für ben 29 en Januar 1789 in Mufik feşen tỏnnte; er Gabe daju ein gutes Sủjet, nur bảte er fid jur Bearbeitung Depelben hinlånglidje seit aus. Es wurden ibm jects Monate jugeftanden, fo, onf er im laufen: Den Juni:Monate bes folgenden Jabres ganj mit feinem Stúde fertig feun múfte. Er war hie: mit fo jufrieben, Daf ex verficherte: eine fo lange Beit wàre mebr, als er verlangte. Slurg Darauf

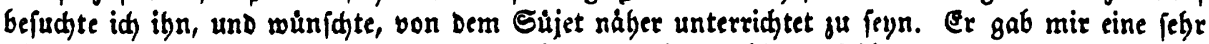
idjon geidriebene Bejhidte ber Alfsol (Dies war fein gemábltes Süjet) ju lejen. Id lap fie, und fand das Sưjet ju einer Dper faft ju tragifd, aber boller \&eben uno Intereje, und war ùber= jeugt, Das foldjes, von einen geididten Didter bearbeitet, ein trefflidyes Singftúct geben múste.

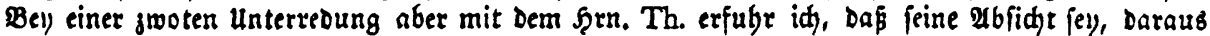
ein Drama in 5 aften ju maden, worin nur bas gefungen werDen pollte, was eigentlid in ber Bejhidhte felbft, als fingbar, vortommt. Idh hatte nidhts gegen eine foldje Bearbeitung biejes Sújets einfuwenden; fie ift vielleidgt Die ihm am meiften angemesene: id tonnte aber nidgt um:

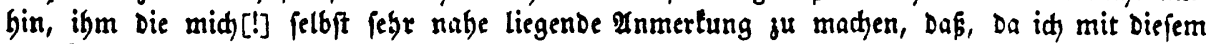

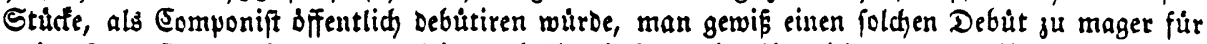

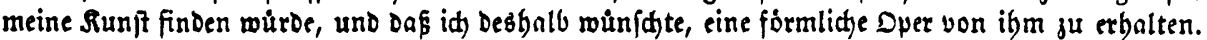

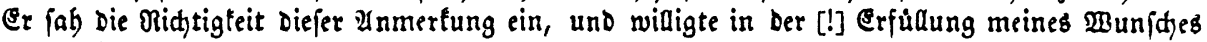
um io lieber, da es ifm, nach feiner eigenen Bemertung, weit leidhter werden wúrde, aus diejem Sûjet eine tưrgere Dyer in $3 \mathfrak{A}$ ften, als ein langes Drama in 5 dEten, ju verfertigen. Wir waren bierúber nun wieder ganz eins.

Einige 3eit nadbber, nadjoem Die Direction von Der $\mathfrak{B e r b i n d u n g ~ D e s ~ S ̧ r n . ~ T h . ~ u n t e r r i d t e t ~}$ war, wúnidte fie, Den plan bes Stưt's yon ifm ju erbalten, um io wol den siang befielben fennen zu lernen, als aud bey) seiten auf Die Deforationen, Mafdinerien, Sleidungen u[w., die Darin vorfommen wúrden, bedadjt jênn ju lónnen. Nur nach múbjamen úberredungstúnften gelang es mir, Die Erfúalung diejes ganz natưrlichen Berlangens Der Direction gu erbalten. Mir felbft war um fo viel melor Daran gelegen, weil id yon feinem plane von ibm felbft, bis dabin, nody nidhts beftimtes erfabren batte, und bey) diefer selegenbeit mit einemmale von dem ganjen Stúcte unterridtet gu werden glaubte. Der Plan erfdien endlid, aber wie? Er war in 5 aften abgetheilt, ftatt Daj unire vernbredete Dper, wie gewobnlich, nur 3 ufte entbalten folte; er war alfo noch Der alte plan feiner erften Joee eines Drama; im úbrigen entbielt er, wie joldhes allen Mitgliedern ter Direstion nod befannt feun wird, eine blofe troffene angabe Der in Dem Etúfé yortommenden Decorationen: Bon Dem Eange Des Etúfes fel6jt war Darin nidht Die Diede. In: Def́en erfannte Die Direction Dod) Daraus, Daf in $\mathfrak{A}$ fiid)t Der Decorationen Barin nidjts unaus: fủbrbares vorkảme, und ủberließ̄ nun die Bearbeitung des Etưț́s der weiteren Berabredung swifden bem Şrn. Th. und mir.

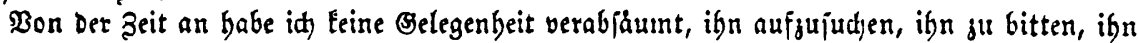

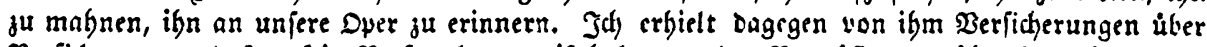

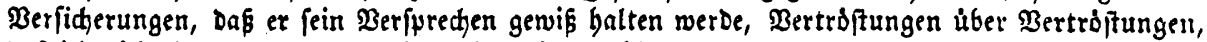

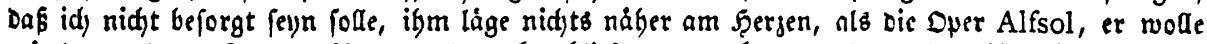
mir ben und Den $\mathfrak{D a g}$ gewis den erften $\mathfrak{A f t}$ abliefern, und Eam ber $\mathfrak{T} a g$, fo erbielt id fatt bes

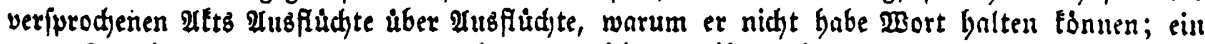
neuer Zermin wurbe feffgefeht, Der wieberum nicht von ifm getalten wurde, und fo nurbe ids yon ifm yon Monat zu Monat, von Bodje zu Bodje, yon einem $\mathfrak{I} n g e$ jum andern bis jum Anfang Des Juni:Monats aufgezogest, ofne nur eine Eiene, nur eine $\mathfrak{\text { Arie }}$ oder Recitativ, ofne nur einen einjigen $\mathfrak{B}$ crs yon Der Alfsol in Şänden ju baben. Th bảtte Doch erwarten follen,

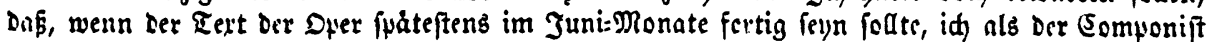
Deffelben, cine peitlang vorber ben erften $\mathfrak{A} f t$, wenigftens einige $\Xi_{i}$ enen Defelben von Dem Didtter erbalten wúrbe, un fo mit ibm jugleich ju arbeiten, und ju red,ter peit mit Der Eompofition

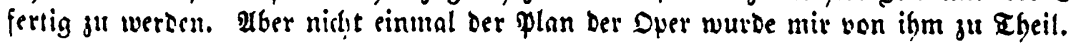


P\}un bielt id mit meinen $\mathfrak{X}$ forbcrungen inne; Denn id) fabe bie Unmoglidyleit ein, Daß aus Diejem Stứfe fừ Den nảdften Rỏnigl. Seburtstag etwas werben tonnte; und Da id glauben mufte, Daß Şr. Th. Eeine Dper für mid, machen wolle, oder lonne, fo that id Darauf Ber: jidjt, uno úberdad)te nun, wie Diejer Manget fo gut, als nioglid, ju erję̧en wáre. Die Direstion, Die foldjes nidbt weniger erwàgte, proponirte mir nun eine Dper jur Eompofition, bie fie im

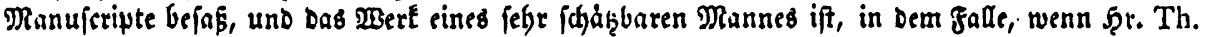

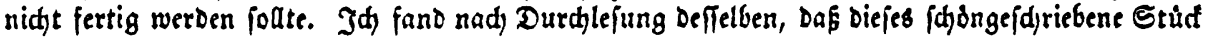
in Dem ३̧ujdnitte feiner Srenen ju jebr bie form bes Drama habe, das nidjt gejungen wird,

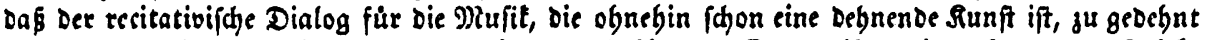
\{ev), und zu jebr an Raisonnement grảnje, Das fứ Den Eomponiften eines fo langen Stúffs Darin teine weitere Mannichfaltigleit fely, als Der übergang vom Necitative jur Irie, und von

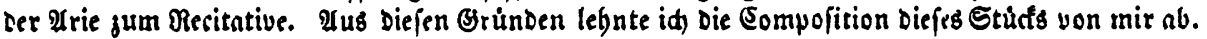

Der Juni:DRonnt gieng nun ju Ende, und $53 x$. Th. wurbe vor Die Direction bejdieden, um feine Dper abjuliefern. Er erídien, und bradte - jwey Erenen Des erften Attz. Die Direction war gưtig genug, ibm î́ren Berdruß

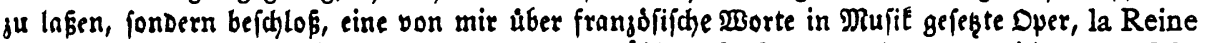
de Golconde, fủr Den nảchiften $29^{n}$ Innunr ju wáblen, fie fognr Durd) Den nemlidjen 5̧rn. Th. überjek̨en gu lafen, und inn jur Bollendung feines angefangenen Stúf's idj riftlid ju engagiren,

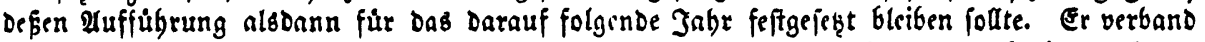

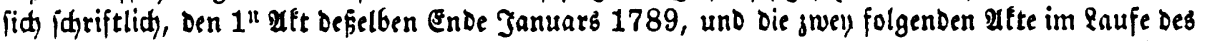
folgenden februarb abjuliefern, mit Der binjugefügten (5laujel, Daf wenn er in biejen Rerminen

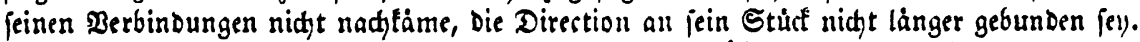

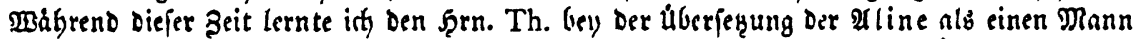

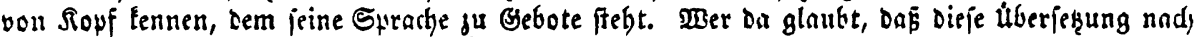

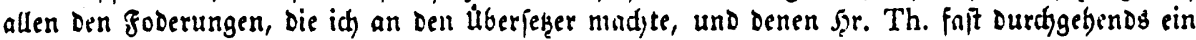

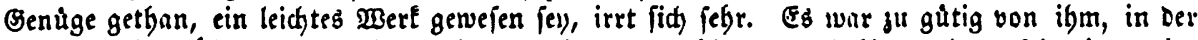

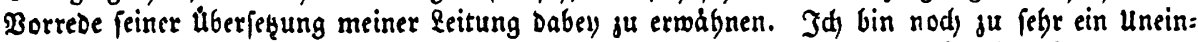
geweibter jeiner Sprache, als bus meine leitung in etwas mebrem bàtte beftefen tónnen, als

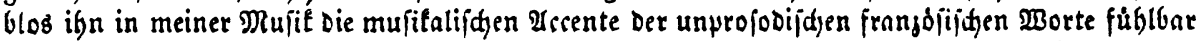
ju machen. Indesen lernte id/ ihn von Der andern Seite auds liebeys wieberum als einen Mann

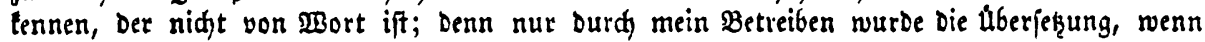

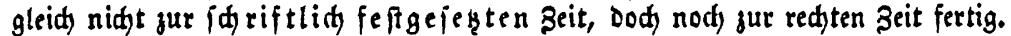

Die Direction felbft war nun Durdy Die Erfahrung ủbergeugt, wie wenig auf Des $5 \mathfrak{2 n}$. Th.

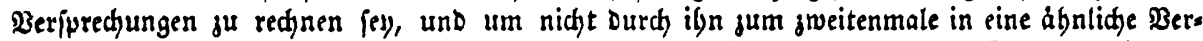

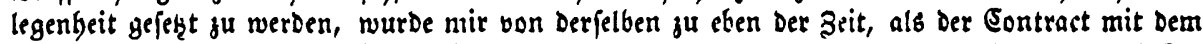
Şrn. Th. gemadt wurbe, einmúthig aufgetragen, unter ber J̧and ben 5̧rn. Baggesen, Defen

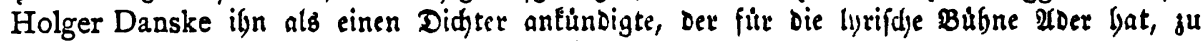

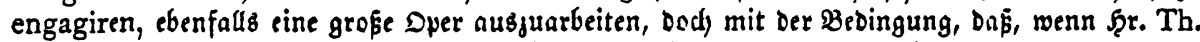

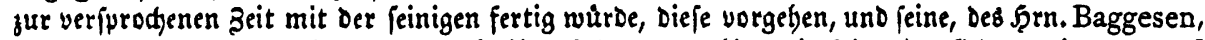

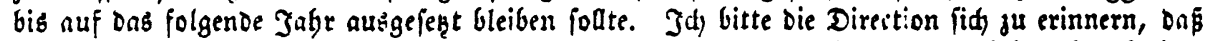
Diejer mir gegebene 2 uftrag urotocolirt ift. J̧r. B. Der Damals mit andern $\mathfrak{A}$ rbeiten bejdåftiget war, veriprach bierúber nichts gemiffes, fd)lug es aber audh nidht aus und war úbrigens mit ber

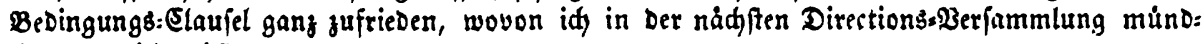
lidjen Beridht abjtattete.

Nun fängt eine neue Epodje abermaliger Erwartungen von meiner Seite, und Bergogerungen

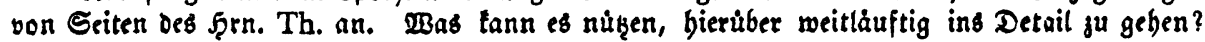
Rurr, Der Januar fam beran, ofne Daj ich von Der Alfsol mebr erfubr, als vorber. Bey Den baufigen proben ber Aline, Die im Januar gebalten wurben, hatte idt Eelegengeit, ben f̧rn. Th.

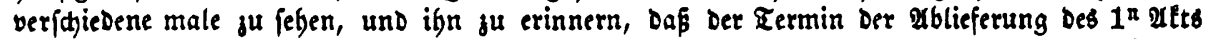
feiner Dper berannabe; er Gatte beftándig eine von feinen vertroffenden und veriprechenden lurgen

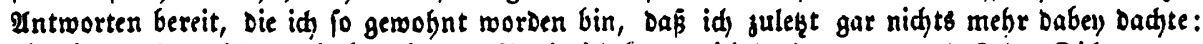

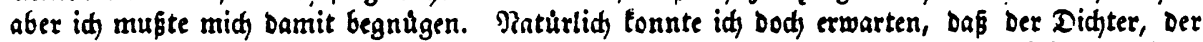
fid) mit bem Eomponiften verbindet, um ein lyrifdjes Etưf auf Die Búbne ju bringen, eineı Drang in fidh füblen wúrbe, mit ibm úber bie Bearbeitung bejelben ju fpreçę, ibm ben plan 
ju Detailliren, ifgm ju jeigen, was er fertig habe, uno jeine Resynung Darúber ju vernebmen. Nidhts von allen bem wahrend biejer ganjen seit, in weldjer ber $1^{e}$ Utt idon fertig fenn follte. 3̂weifel und Unrube bemádjtigten fidy meiner. Id tonnte nidyt obne Grauen Daran Denten, Dap id) Durdy bic blope Radlápigleit ober Das unvermogen eines Mannes, tem bie Direction mit ibrem Bertrauen beehrte, vielleidgt jum jweytenmale aufgesogen werben tonnte. Da id bey Die: fem Gedanten meine Sủnfter= E⿹ Drang auf Das flebentlidffe in ifn, er modjte bey fo bewanden umftänden, als ein freund fúr mid bedadyt (e)n, meine Noth fu Şerjen nehmen, unb auf eine Dper für midh Denfen.

Benn biebey) Eabale, ein wort, Das id jeşt fo oft boren mus, gewejen ift, jo geftebe id, Daß nur id allein Die Eabale geipielt habe, ob id gleid bibber Das $\mathfrak{B}$ Bort nur Dem Namen nad gefannt habe. In allen Berbalttniffen meines ganjen lebens babe id fo wenig vermertt, das Eabalen gegen mid gefpielt wảren, als id gegen andre gejpielt bätte. Diefe war menigftens eine Durd Die Direction felbft authorifirte Eabale.

Şr. Baggesen war to weit Davon entfernt, Eabale gegen Den Şrn. Th. Ju madhen, bas er mid) [!] vielmebr auf alle SBeife meine furdjt unb şweifel in $\mathfrak{A} 6$ fidjt ber Alfsol ju benehmen fudhte.

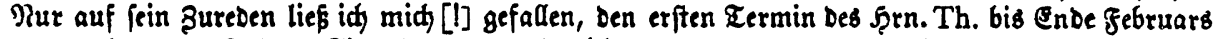
auófubebnen. "Sodten Sie alsbann nod nidgts yon Dem Şrn. Th. erbalten baben", feste ex binju, "To veripredje id, Sie nidjt im Stidje zu lajen. Jdy werbe nun auf ein gutes Sújet Denten; aber Sie mùfen mir IGre J̧and Darauf geben, Das Sie, wenn mein Stúd fertig ift, und Sie es componirbar finden, es als cin Eefdent bon meiner freundidaft annebmen, indem id Jhnen ùberlafe, weldyen Bebraudy Sie Davon machen wollen ober tỏnnen." Jd verftand und

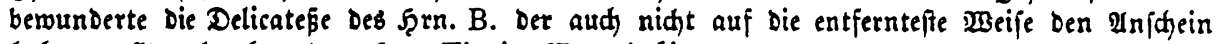
baben wollte, als ob er bem Şrn. Th. im wege [!] táme.

Th veriprach gern alles, und war in fo fern berubigt, als id nun auf eine ober bie andre Beife fidjer auf ein Etúct Redjnung madjen tönnte, uno nur ber einjige unangenehme fall für

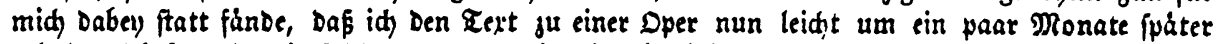
erhalten Dưrfte, als mir foldjes von ber Direction bewilaigt worben ift. Indefien wolle id diefe Aufopferung meiner z̧eit unb Erholung im Sommer mir biejes Jabr gerne gefallen lapen.

In Diejer Berubigung erwartete id nun vollends Den $\mathfrak{X}$ uzgang Dez Januarb. Er ging vor: über; und Şr. Th. hatte nidhts für mid fertig. Id wurbe um biefe zeit frant, und veridjiebene=

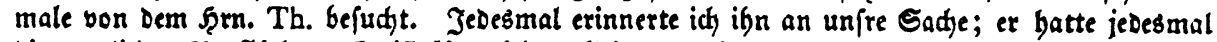

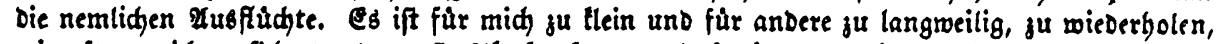

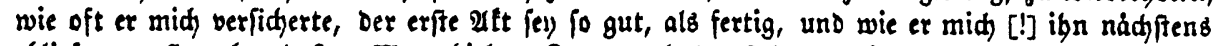
abliefern molle, obne bas er Bort hielt. Surg, audy ber februar gieng ju Ende, ofne das idh einen Edyritt weiter mit ifm war, als Das ganje Jabr vorber.

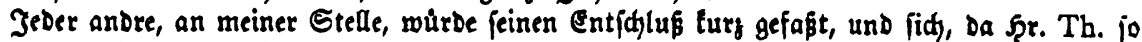
wenig Wort bielt, fdjon längft von ifm getrennt haben. Bas tonnte midh Denn fo fandbaft an ibn [!] fefthalten? Swely ober breverley Betradtungen. Die erfte: Th. if ein Mann von fdjąs:

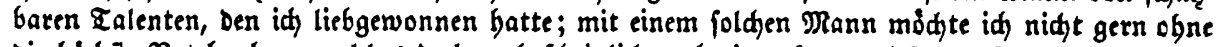
bie bodpite Not bredjen, weldyes Doch wabrideinlid, nady einer fo gewaltjamen Irennung exfolgt jeyn wúrbe. Die jwelste: Th. Gat viele freunde, bie um alles wusten, beren Borwúrfe er fid ausfekzen modjte, wenn Durd feine Sduld jum jweytenmale nidhts aus Der Dper Alfsol gewor= Den ware, wovon idjon ein Jabr yorher Die Rede geweisn war. Nod ein britter Brund war bel)

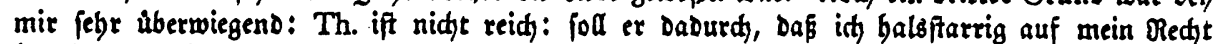
beftebe, einer tleinen Einnabme entbebren, worauf er bielleidht idjon geredjnet baben tann? WRein Entidlus war Daber feft geblieben, Den ganjen februar nod) abjuwarten, ob id gleid eigentlid bieju lein formlidjes Redtt hatte; Denn nur Die Direction, mit meldjer er contrabiret batte, fonnte igm die Berlángerung Der Eermine jugefteben. Indefen war id úberjeugt, Daßs fie bier= úber [nid,t] pointialeen wurbe, Da nur id Gauptiảdlid, babel) fu gewinnen ober fu verlieren batte.

WBáhrend biefer ß̧eit, es war nadh einer der erften Borfteaungen Der $\mathfrak{A}$ line, tam Şr. Baggesen,

Dem [!] Die Yuffübrung Diefer Dper mit einemmale ganj jum EGeaterdidter begeiftert hatte ju

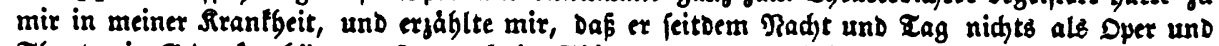

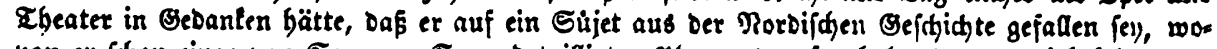
von ex fhon cinen von Scene ju Scene betaillitten plan entworfen habe, Den ex mitbradjte, um

Ardio für Mufifitififenídaft 
ıneine Meynung Daruber ju erfahren. Die überjdyrift war: Erik Eiegod, ober Die Einnabme von Julin. Ih ertannte fijon in bem plane, ber fo gang nad meinem Sinne war, ben Dichter, Der von feinem Sujjet begriftert ift, ber gleid in ber exfen Scene Den subjrer mitten in Die

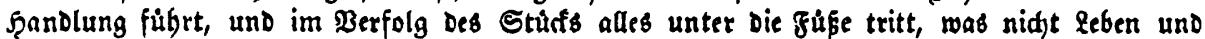
Bårme athmet, Der Die Bedúrfniffe Der Expofition in Şandlung und Empfindung zu verwandeln verfteht, und ber bem Sornponiften Egaraftere und Situntionen jubereitet, bie fưr bie Mufit gefchaffen find. Id) bat ifn, er modjte nad Diefem Plane Das Stưf ausarbeiten, und es mir, unfrer $\mathfrak{B e r a b r e d u n g ~ g e m a ́ s , ~ u ́ b e r g e b e n . ~ E r ~ b r a d t e ~ m i r ~ e i n i g e ~ T a g e ~ b u r a u f ~ c i n ~ p a a r ~ E c e n e n ~ b e s ~}$ Infangs, Die meiner Erwartung vdlig entfpradjen, Dann wiebcr etwas, und wieder etwas, and

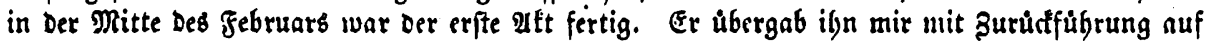

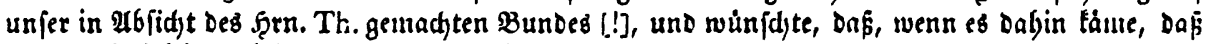

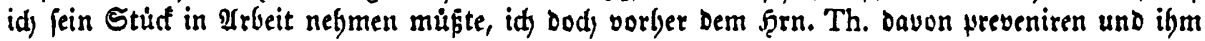

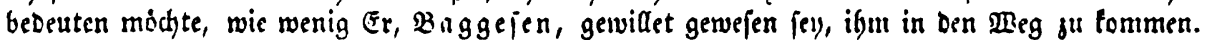

Da nun tis Ende Februars Şr. Th. midh yon einem Tnge jum andern aufgebalten hatte, und fưr midh feine Seit mebr zu verlieren war, wenn idy felbft bey meiner $\mathfrak{A} r b e i t$ midh nicht ábers eilen jodte, io mujte id nun Ernft gebraudhen. Id) beflagte midh Demnady am Jrentage, Den $27^{n}$ Febr. in Der Direstions:Berjamtung bitterlich ùber ben $\mathfrak{5}$ rn. Th., Der unich nun wieberum einen ganjen Rowat hàtte verlieren lajen, und erwábnte nun jum erftenmale dajelbft Der Bag:

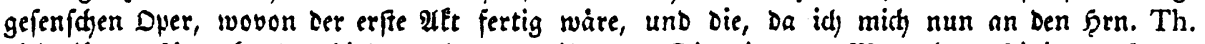
nid)t länger für gebunden hielt, nad) Dem $\mathfrak{B}$ illen Der Direition Den Mangel Des feinigen erjek̨en,

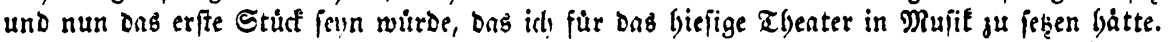

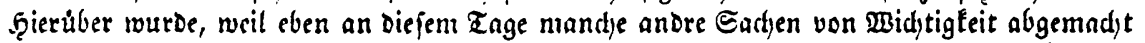

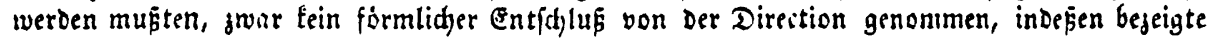
jedermann feinen gerechten Unwiden úber Des Şrn. Th. Unthátigleit und Unftattgaftigleit in

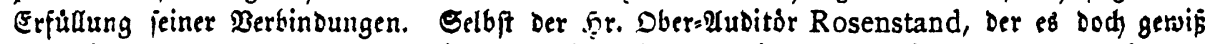
gut mit Dem J̧rn. Th. me!nnt, verbarg Darúber feinen Unwillen fo wenig, Das er grabe heraus fingte, Das, Da Der Did,ter jeine Berbindung fo wenig erfüat babe, weber bie Direction, nod) idy,

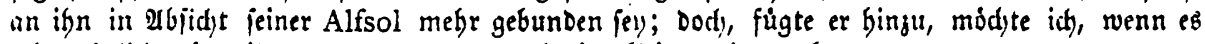
mir móglid måre, ibm, Den Şrn. Th., noch eine Eleine seit jugeben.

Idy glaubte midh nun ùbergetgt, Daf Die Direstion fidh yon ihren Berbindungen mit Dem ñrn. Th. frel) hielt, (wenigftens war fie es nach Dedyt und Bialigfeit) und baj es einjig und allein yon mir abfieng, of id nod) eine zeitlang bie ablieferung feiner Alfsol erwarten wolle,

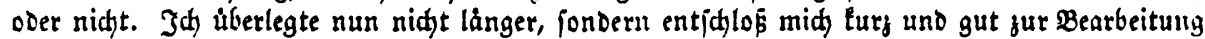

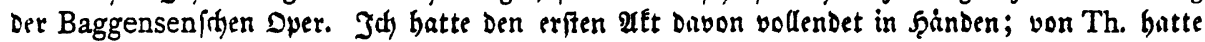
id) nidhts, uno war ungewis, je etwas yon ibm gu ergnalten. Baggefens over war nad) meinem Einne; Th. Eonnte mir vielleidyt eine eben fo gute, vielleidht eine befire, vielleidyt aber auth eine id)ledtre liefern. Wis war bier lange fu úberlegen, Dem gewißßen \&uten bem [!] ungewisen Bepren oder Sdjledtren vorjujieben? Mein Entidglus war bemnady gefast, und id nahm mir yor, joldjes Dem Şrn. Th. jelbft fdriftlid betannt gu madjen.

Aber an Dem nemlidyen Eage, Des $\mathfrak{A} b e n d s$ nad ber $\mathfrak{B o r f t e l l u n g}$ ber alline, verlangte Şr. Th. eine Unterredung mit mir auf Dem Egeater. Id tonnte es nidht unterlasen, mid) gegen ihn mit Bitterkeit ùber fein Berfahren mit mir ju befllagen, und madte ihm nun gerabe heraus befinnt, unter welden Bebingungen B. eine Dper für midh gemacht babe, zu welder er burd) mid yon

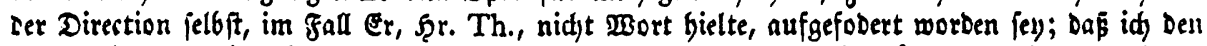

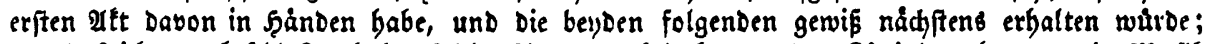

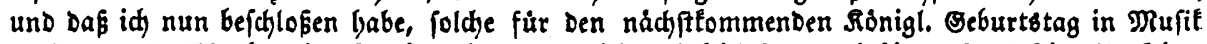

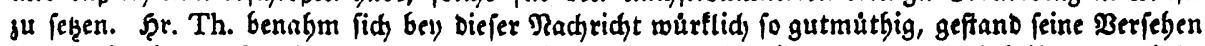
fo offengergig, verfurady aufs neue fo ebrlid, uno jprad mir von bem Madtheile, ben biejer

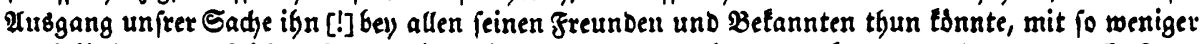
surưdfigaltung, Das id aufs neue in meinem Entidlufe wieber wantend gemadtt wurbe. Er fagte

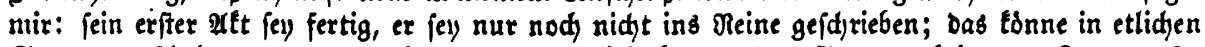
Stunden gefdeben; ex wolle mir folden Den nadjittommenden Sonntag Gringen. Rurs, unire

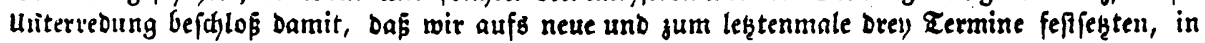
weldjen id) gu bem endlidjen Befizęe ber unendlidjen Alfsol gelangen foule. Der nàdyfe Eonntug 


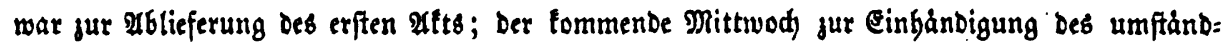
lidjen plans vom $2^{n}$ und $3^{n}$ Ilt, (Denn id war nod immer fo univisend uber ben plan feines

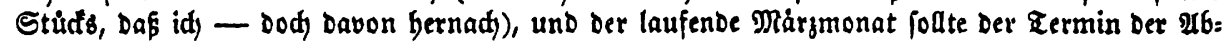
lieferung ber besben lesten fertigen Atte fes)n. Er verlangte von mir, baj, sbenn er biefe 3 Eer:

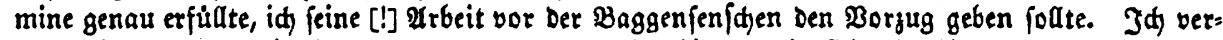
jprach ing joldhes mit einem Szandichlag, jeşte aber binju, wie fid obnebin bon felbft verftebt,

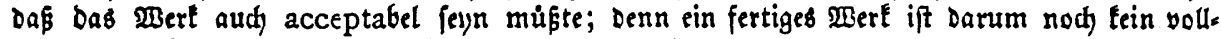
endetes Bert. Und jo jajeden wir fegr freundidjaftlid.

J̧x. Baggensen, ber Den nemlidjen $\mathfrak{A} 6$ end ebenfalls auf Dem gheater war, und unfre Unter: rebung von ferne geíeben batte, blieb, weil er leidjt rathen tonnte, wovon bie Biebe wáre, um beym $\mathfrak{B e g g e b e n}$ Den Erfolg Derjelben von mir ju erfabren. Th jagte ibm grabebin, was aufs

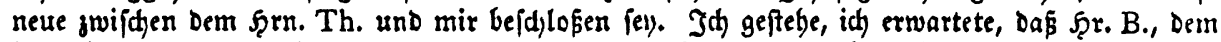
id) etlidge Stunden vorber geiprodjen und ibm gefagt hatte, Daf es bely mir nun ganz ausgemadyt (e)), Das id Den Erik Eiegod in Mufit jeţen würde, nun wenigftens einige unjufriebene Morte

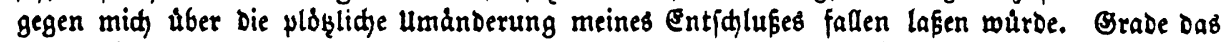

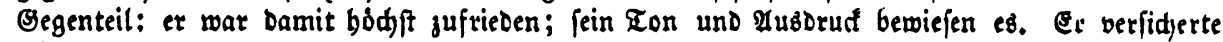
mid, Das er bels Diejer Madjrid, mehr Rube uub freube empfánde, als bels ber ihm juvor gege: benen, weil es ifm unrube gemadt baben wưrbe, Dap for. Th. Doch immer nod hátte glauben tonnen, ex habe ifm im [!] B̉ege fommen obcr verdrảngen wollen. Er fehte binju, Daß ex nun mit weit mehr $\& u f f$, sufriebenbeit und Muffe an ber $\mathfrak{B o l l e n d u n g ~ f e i n e s ~ E r i k ~ E i e g o d ~ a r b e i t e n ~}$

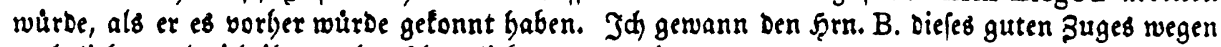
nod) lieber, als id ifn vorber fhon liebgewonnen batte, und wurde von biefem alugenblicfe an fein wabrer und wàrmfter freund.

Die Şerren, bie allenthalben Eabale finden, mögen mir body erflåren, welche boje Abjicht 5nr. B. und id in biejem 2ugenbliffe gebabt baben. Dody vielleidt wird man bas alles nidht glauben, oder gar Das Gegenteil glauben. Meine Sadje ifit aber nidjt, bier ju unter[uchen, was geglaubt ober nidjt geglaubt wirb, jondern ju ergíblen, waz gefdeben ift.

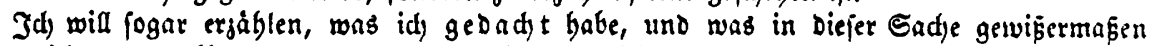

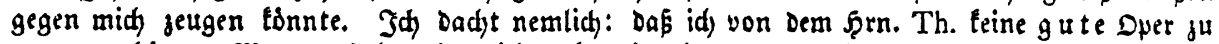

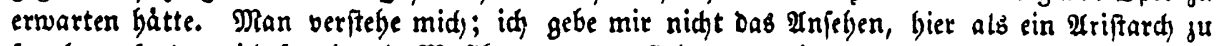
ipredjen, fondern ich fpreche als $\mathfrak{M u f i t u s , ~ u n d ~ v e r f i t e b e ~ u n t e r ~ e i n e r ~ g u t e n ~ D p e r ~ f o l d j e , ~ b i e ~ m u f i = ~}$

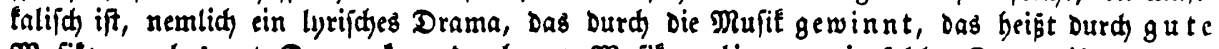
Mufil: mandjes gute Drama fann Durch gute MufiE verlieren; ein foldjes Drama if entreder nicht Dper, ober wenigftens teine gute Dper. Der Grund, warum idy fo von Dem Sprn. Th. Dadjte, lag Darin, Daß id in allem, was mir yon ibm, felbft von einigen fertigen Etellen ber Alfsol, betannt geworden war, mebr einen ftalken, Eraftwollen, Eritifdyen Dichter bemerf́t batte, als einen Didter, Dem Die Spradje Der Empfindung ju Bebote ftunde, Die nur Die einzig wabre und gute Spratje Der Dper ift.

Th) Dad)te ferner: Daf ungeadtet feines abermaligen Beripredjens die Dyer Alfsol entweber Dod, nidht im Mảrz, ober Dod, als ein febr unvollendetes Stúd fertig werden wúrbe. Şiegu hatte id) nun wieder gute Bründe. Id) war nemlid) einige seit vor unfrer lek̨ten Unterredung bey ingm gewejen, um bod endlid, etwas yon Alfsol ju exfabren. Nadjoem er mir einige einjelne fertige Stellen aus einjelnen Scenen Des erften 2 fits vorgelefen hatte, Die id, weil idy von Dem 3ujam: menbange Derfelben nidjt unterridjtet wax, nur bunfel fáste, bat idh ihn, er módjte mir oodj end:

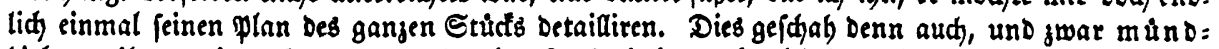
lid, weil er, wie ex fagte, Den plan im Sopfe habe. Aber id bentertte bald, wie ungewis er Darin nod, mit fid felber war. Dies erbellet aud baraus febr beutlid, Daj er midh [!] etlidje Eage nadber lagte: er fábe fid genóthiget, Dem Stùde 4 dfte gu geben; wovon Dody nie vorber Die Oede gewejen war. Bel) unirer lę̧ten unterredung aber auf Dem Theater ertlärte er mir wieber, Daf es Dod) bel) 3 atten jein Bewenden behalten wurde, fúgte aber bie frage binju, of

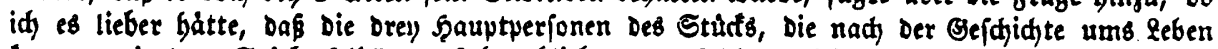
tommen, in bem Stüde felbit am Reben blieben, um foldes frolid ju bejhliefen? worauf ich blos erwiberte, baj ex ja am beften wiß́pen múse, wie er feinen plan angelegt babe. Jdy úber: lape einen [!] jeben, ber fiil] einen 2 ugenblid in meine Stelle feşen will, fu urtbeilen, weldjes 


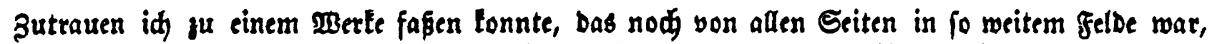
worauf 5 r. Th. idon fo lange seit jugebradjt hatte, und Ins nun binnen einem Ronate vod: endet Daftegn folte. Entweder ift Die Berfertigung einer Dper etwas fo leidtes, Das in turjer Beit gemadjt ift: und Dann war es unverjeiglidy von Dem Şrn. Th., fie nidjt fhon längft ab:

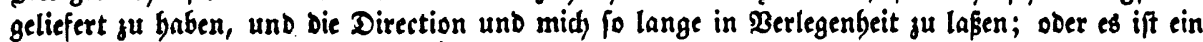
idjweres Sunftwert, Das seit und ûberlegung erfobert: in biejem falle tonnte id, Da Şr. Th. in fo langer seit nur fo wenig Davon gemadht batte, und felfft nody nidgt mit bem plane einig war, wol idwerlid erwarten, es in vier Bodjen vollendet von ifm ju erbalten. Und po wars. Ih

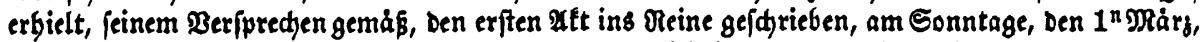

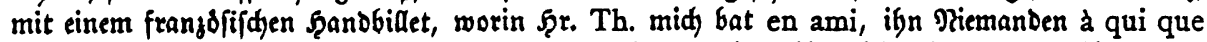

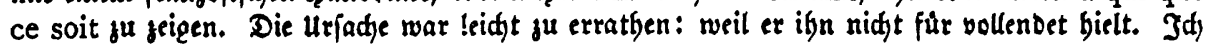
las ifn, und fand, was ich erwartet batte. Die Direstion mag úber ben \&ang bes erften afts Diejer Dper jelffit urteilen.

Die erjte Scene ftellte eine bey einem Norbijdyen ₹empel gefeiligte Begend vor. Ein priefter.

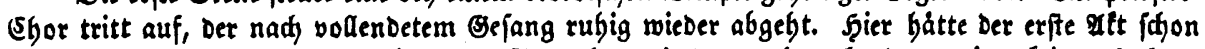
wieber fhliefen tónnen, Denn bie jwote Siene hat mit Der yorbergehenden weiter Eeinen sujam: menbang, als Die unverảnderte Decoration. Nun tritt Alfsol, Die Şauptperion bes Stùt's, auf, allein, ofne alle Borbereitung, wodurd fie bem subbrer hảtte interefant gemadtt merden lónnen, aber audh ofne etwas ju fagen, woburds fie fid ibm interejant machte, benn fie befingt - bie liebe fum $\mathfrak{B a t e r l a n d e : ~ l a l t e s ~} €$ üjet in Dem Munde eines jungen frauenzimmers. Sie wirb in

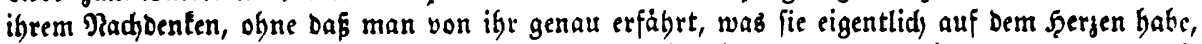
yon ifrem $B$ ruber unterbrodjen. Die Dritte Siene beftelot demnach aus einer Unterredung zri: fhen Bruber und Schwefter: Eann man in einer Dper jwely fáltere Personnagen auf Der Báthne jeben? Und diefer Bruber, was hat er interesantes vorjubringen? er fucht feine Sdjwefter für feinen freund Harald, Der fie liebt, jur Begenliebe ju benegen. Bejer wárs Dod), Daß Harald felbft fid Darum bewưrbe; aber ber Bruber will erft von ber Schwejter erfahren, ob fein Befud)

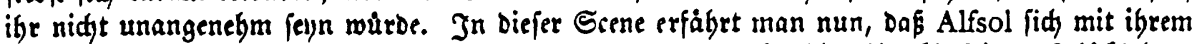

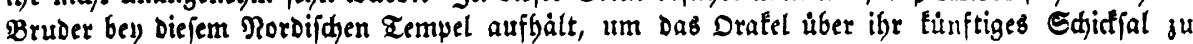
befragen. Der Bruber verläft fie, um feinen freund jelbjt ju bolen. Alfsol, wieder allein in ber vierten Scene, áfipert in einem zweiten Monolog einige Unrube úber ben naben Bejư Haralds. In Der $5^{\mathfrak{n}}$ Scene $l$ ldmmt ibr $\mathfrak{B}$ ruber in Begleitung Des Harald wieder, Der fidy nun felbft um Alfsols liebe bemirbt, aber weber Jin nod Rein erbảlt, weil alles yon bem bevorftebenden Draḱelipruche abbången wird. Alfsol wirb von einem igrer Mäbdjen abgerufen, um in ben Eempel ju geben, wo alles jur Oratel:Seremonie fertig ift. Jhe Bruber uno Harald bleiben in ber $6^{\mathfrak{n}}$ Ecene, die eine lange freund fdjafts: Scene ift, allein, und idyodren in einem Duett fid ewige freundidjaft. Şeipen bas DperneScenen, bie gejungen werben foden? Jit bier ber geringfie theatralifde Effett?

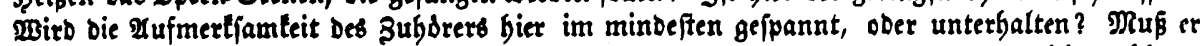
nidgt vielmehr, wenn bas Dehnende ber Mufił bingulsnumt, er mag wollen ober nidt, gáfgnen und einfidlafen? - Die Deioration verwandelt fids nun in bem [!] Inneren bes Eempels, worin bie Dratel-Eeremonie vor fidh geht, und Der Dunkle Sprud ufber Alfsols tünftiges Edjidfal ver:

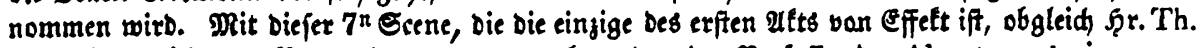
bel) weitem nidjt Den Nub̨en Daraus gejogen hat, Den ber Berfaffer ber Alceste aus einer gand

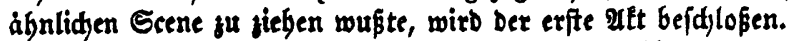

5̧r. Th. Gatte mir immer gejagt, Daß Das Sujjet Der Alfsol voller Incidens fey, und dap

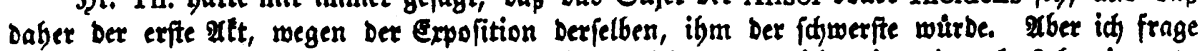
einen jeben, was benn hier eigentlid exponirt ift? Nidgts, was nidgt mit weit mebr keben in jwey) Erenen batte exponirt; ja id mddte faft begaupten, ohne alle Expofition batte voraubgeję̧t wer:

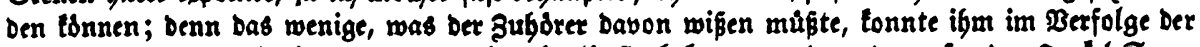
f̧anblung, und burdd bie ફ̧andlung felbft, bes)laufig betannt werben, ba aufer ber Drafel: Scene alle vorbergebenden von gar teinem Belange, nod) Jnterefe, für ihn finb. úber bie sálte ber Situationen will id, nidgts weiter fagen; jebweber extennt foldje von felbft. ulbrigens geftefe id) gern, in Dem Dialog. Des Şrn. Th. mandje idjongejagte Stellen, tỏnenbe Berje, eine tráftige Epradje, leidjte Berfification u. ogl. bemertt ju haben; alles bas tonnte man von einem Manne, wie Şr. Th., erwarten. A6er Das finb bel) weitem nidjt bie Şaupteigenjdjaften einer guten D per; 
Fondern Wåme und Leben Der Empfindung und Şandlung, theatralijder Effeft, Situationen, Die Aufmerffamkeit verbienen, und wobey eben fo wol, als in jebem andern Drama, Degelmápig:

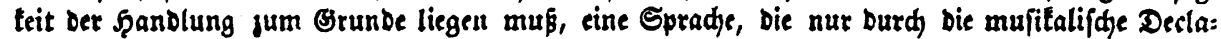

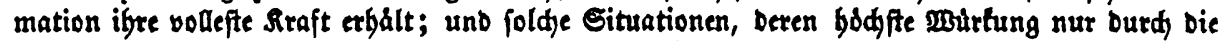
vereinte eewalt Der Inftrumental: und $\mathfrak{B}$ ocal:Mufil mit Der Poefie erfalten wirb; Die folglid immer nur in Rudffidgt auf Die begleitende Mufif von bem Didjter gridjaffen find, und bie obne Mufit entweber unausfübrbar bleiben, ober bod allen Reiz̨ verlieren würben. Das find bie f̧aupt: eigenjigaften einer guten Dper: Daburdy wirb fie ju einem für fid beftebenden Sunftwerte, Das, fowie das Eraueripiel und jebes andere Sunftwerl für Den Didgter fo wol als für Den Eomponiften,

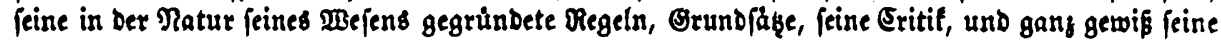
gropen Sdywierigkeiten Gat; Das eben Deswegen eine Slippe ift, woran fo mandje grope Didter und Eomponiften, Die fid einbildeten, jebes Drama, Das gefungen wurbe, fes) eine Dper, gefheitert

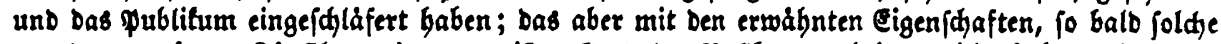

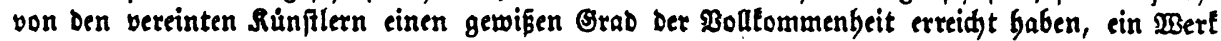
Des Beiftes unb Des Bejdymades ift, bas Den exften Oiang unter bramatifien Sunftwerten be: Gauptet, Deffen Bủrtung auf Dhr und Şer nod tein publitum hat wiberftehen tonnen, und Das

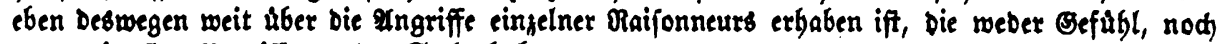
Den geringften Begriff yon ber Eadje haben.

Bon Diefen Eigenidjaften war in Dem exfen aft Der Alfsol bes Şrn. Th. faft Eeine Epur ju

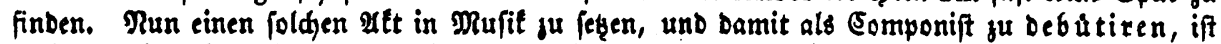

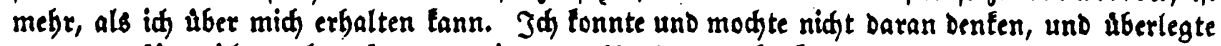
nun, was fưr midh ju thun (e), um mit guter $\mathfrak{A}$ rt Davon abjutommen.

Str. Th. Gradte mir nun aud) veriprodjenermajen ben Plan bes $2^{n}$ und $3^{n}$ afts. Jd er:

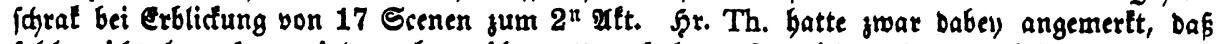
foldje nidjt lang (es)n wưrben; bey nảberer unterfudjung fand id dods, Das viele Esenen, bie zwar nur turje âberidriften hatten, als f. B. Sie berathidjlagen fid, was zu thun (el)

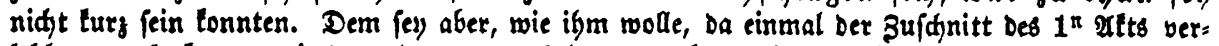
feblt war, fo lonnte mir ber plan ju ben folgenden Alten, bie auf Diejem gebaut waren, nidyts

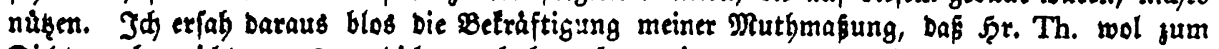
Didhter, aber nidft jum Dpernbidter gebohren feyn moge.

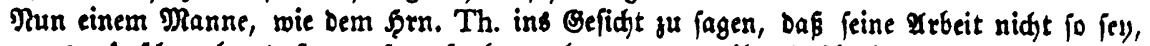

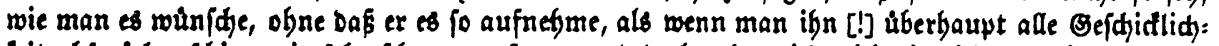

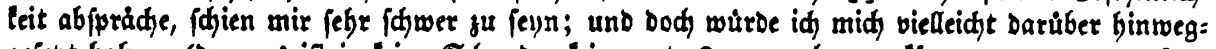
geję̧t baben, (benn es ift ja leine Sdjande, leine gute Oper madjen ju tonnen, wenn man lon: ftige Berbienfte hat) wenn mir bie Bewegungsgrunde, bie id oben fidon angefübrt babe, nidht beftandig vorgefdjwebt und midy ermuntert hadten, bierin mit aller móglidjen Behutfamteit und Delicatefe gu verfabren. Ih teilte ihm nun einige Bebenten úber bieje ober jene Stelle mit, worin er mir $\mathfrak{B e l y f a \mathfrak { l }}$ gab, und fie gu ánbern berfprad. Th wurbe nun breifter, unb fagte inm,

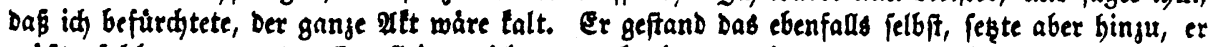
wúpte foldhes wegen ber Expofition nidjt gut abjuảnbern; indeßen mödjte id nur alles wohl úberlegen, und igm meine Joeen mittheilen, ex wolle gern alles fo verändern und bearbeiten, wie idy es wünjaje. Uno jo verließs er midy.

3d) war nun in Dex grosten Berlegenheit. Man tann in Berfen ber Sunft leidt finden, was nidjt befriebigend ift, unb was berknbert werben múste; es iff aber áujerft idjwer, anjugeben wie foldjes berảnbert werben múpte. Sionnte id bas bel) ber Opernbidterey, fo wáre id aud) felbft Didhter, und braudyte blos einen Dảnifden überję̧er ober Bearbeiter meiner eignen Joeen.

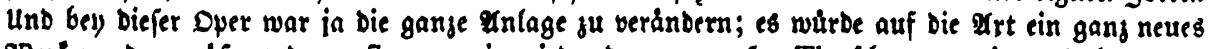
2Bert werben múfen: Dann fiengen wir wieber ba an, wo Ş̧. Th. iđjon yor einem Jabre war; wann batte idh nun wieber bas Enbe feiner 2rebeit erwarten follen? Es if wahr, wenn idh un: reblid batte bandeln wollen, fo batte id, es darauf antommen lafen fosnnen, ob er in ben laufe Des Márgmonats bely allen Berảnderungen und umarbeitungen mit bem ganjen Etứfe bátte fertig werben tonnen. Lảnger war idh ja body nufs allerbodhfte nidft an ifn gebunden. Uaber bas war unmoglich ju erwarten, wenn er ben ganjen plan aufgeben, neu erbenten uno ben $1^{\prime \prime}$ fertigen Aft verwerfen fodte. Folglid) mufte id, biefe unnủbze [!] Berfdyendung feiner peit und Arbeit ju: 


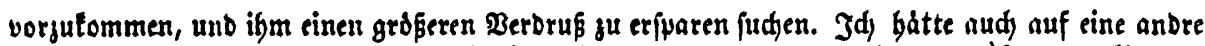
Art leidt aus biejem Bebrange, worin id mit Dem Şrn. Th. gerathen war, 'fommen tosnnen, wenn id fein Manufeript genommen, es Der Direstion vorgelegt, meine Eründe gegen bie Eom: pofition Defelben angegeben, und es ihrer entidjeibung ùberlafien bätte, auf weldye art fie fich mit bem Sprn. Th. aus einander feşen wolle. Şiegu hatte er mid) [!] aber eines Egeils felbft bie Şande gebunden, indem er von mir verlangt hatte, es feinem Menidjen à qui que ce soit ju

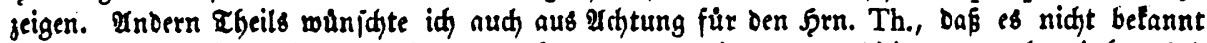
werben moge, wie wenig er in biefem $\mathfrak{B e r f e} \mathfrak{A n}$ lage ju einem Dperndidter verrathen babe. Id

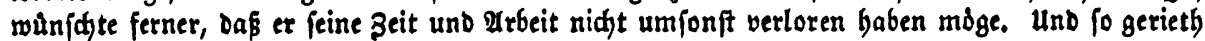
id) auf ben Einfah, ifm einen Brief ju fdgreiben, worin id Das Sûjet Alfsol als zur Dper ganz untauglid, finden, und ign bann bitten wollte, Die Joee, Daraus eine Dper ju madjen, fahren ju lasen, fondern vielmebr Darnus ein Drama nady feiner erften Joee, bie wưtllid feine \&ieblings: ibee gewefen war, ju madjen, indem id) midh nufridtig erbote, alle mufifalifde Scenen barin mit

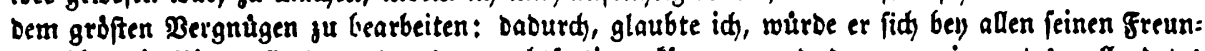
Den úber Die Mid)twollendung ber Dper rechtfertigen lonnen, und Dagegen ein gutes vollendetes Drnma liefern, Das ifm Ehre und Sthabloshaltung fưr bie bely Der angefangenen Arbeit ver: lobrme peit einbringen-múrbe. Delicater, glaube id, tann man fid in einer foldjen lage ber

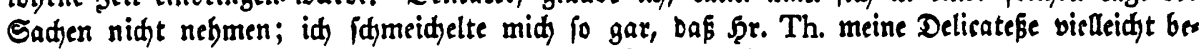
merfen, und mid' [!] in feinem Şerzen Dafưr Dant jagen wưrbe.

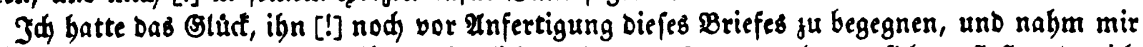

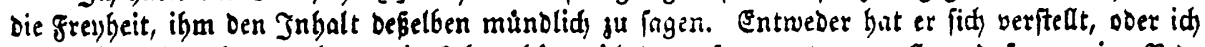
habe mid febr geirret, aber mein leben hảtte id, Darauf verwetten wollen, Daj er meine Rebe fo aufgenommen hảtte, als id es nur immer wúnjdjen lonnte. Wir fdjeben, wie bie bejten freunde, aus einander, und id veriprad, ifm bes folgenden sages alles, was idy von ihm in Şanden batte, jurüfjufdhirfen, und einen Brief beysulegen, befien Jnhalt er jwar idjon wife, aber wovon er, wo er es für nòtig fảnbe, jeben geliebigen \&ebraudy madłen tonnte. Er war Damit fehr jufrieben, und bes folgenden Eages erbielt er mit biejem Briefe alles von mir wieber jurùt.".

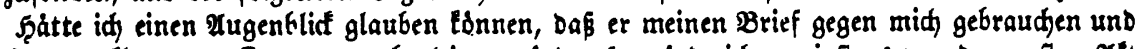
mit einem antlagenden Eommentar begleiten wưrbe, fo wưtbe id, wenigitens von bem erften $\mathfrak{A} t$

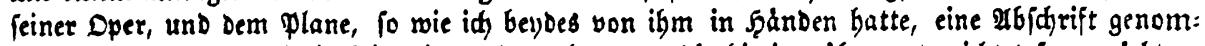
men baben, um fo mol bie Direction, als aud anbre, bie bierin nảber unterridtet feun mòd)ten,

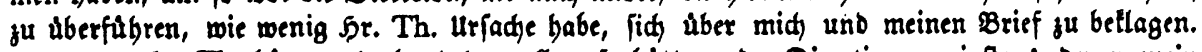
uno wenn 5 r. Th. batte redht bandeln wollen, fo bätte ex Der Direction wenigftens, ber ex mein

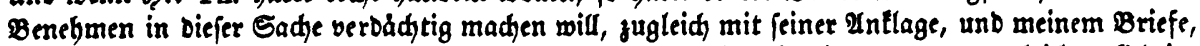
aud Feine arbeit jufdiden follen, Damit fie alles geborig mit einanber ju vergleidjen fid im Stande geieben batte. Seine unidjuld und meine frebelthat wurbe ja baburd in befto bellerem

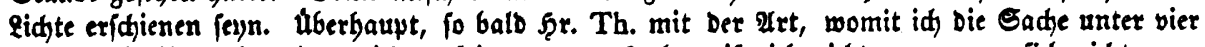
Augen mit ibm abmadte, nidt gufrieben war, io begreife id nidt, warum er fid nidt gand Darưber hinwegieąte, und ber Direction jeine Dper nidjt abliefert, mit weldjer er bod beshalb úbereingetommen ift? Ratưrliderweife ermartete fie foldbe fion lange. Statt bexen aber erbält fie von inm eine feyerlid,e Antündigung, Daßs ex bie bramatifhe Bahn verlafe, und fie nie wieber betreten merbe. Bie reimt fid Das?

Cine Stelle in meinem Briefe an Den Şrn. Th. tann Der Direction auffallen: Die nemlid,

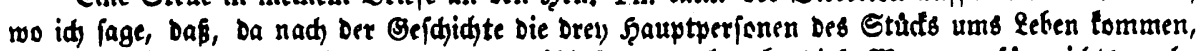

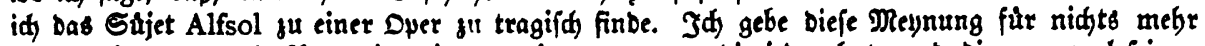
und weniget aus, als für meine eigene Privatmeynung, die jeber bat, und bie man wol feinen Jreunben fagt. Th mutrbe bemohngeadtet, wenn id an einer Dper nur biefe Cataftrophe aus:

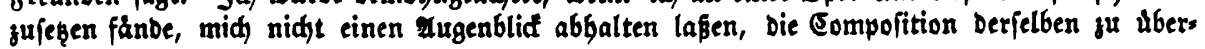
nebmen.

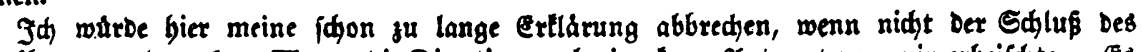
Edjreibens von bem f̧rn. Th. an Die Direstion nod eine turge $\mathfrak{X n t w o r t}$ von mir erbeifhte. "Es wúrbe ganj uno gar gegen fsinen Billen felyn, wenn or feine Dper je aufgeführt fében follte; or wial fie lieber unvollendet lafen, als mich in Die Notbwendigleit gefest feben, jur Eompofition berfelben verbunden ju werben." Dieje Rothwendigleit kann ju feiner Berufigung nie fattfinden. 
Er gebe bem publitum aber sine gute Dper, fo werde id fie, auch wieder. Feinen willen, aus

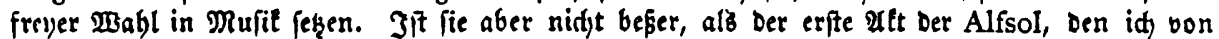
ihm in Szánden gebabt habe, fo gebe id, ifm mein Egrenwort, baf id nie eine feder Darum an: jeşen werbe.

Dies (el) Der Sdhluß meiner Ertlărung. Sie enthălt für bie Direction nidht viel Neues, aber $\mathfrak{B a b r e g}$, und id werde fie, fo bald 5̧r. Th. nod fernerbin (id) bemútgen wird, mid) bels einer Mation, beren 2 d)tung id mir ju erbnlten beftrebe, in gebajfigem [!] Sidjte ju jeşen, Druden

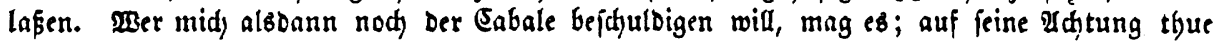
id $\mathfrak{B e r g i d} t$.

Es erbellet ûbrigens aus obiger Ergåblung, Daßs id mid, eines Berjebens gegen Die Direction

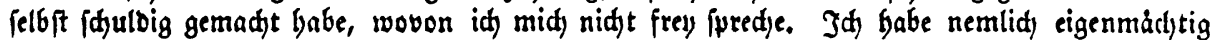
Die verflofenen Eermine, in benen $52 x$. Th. Feine Dper abliefern muste, verlingert. Das id ifm

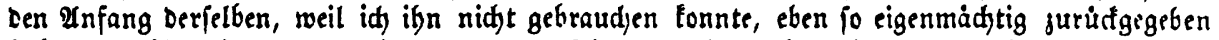
babe, war blos eine folge meines erfiten Berfebens. Id glauste mid) ju bem einen fo gut, als fu bem andern, beredtigt, und bitte fie besfalls um Bergebung.

Die Direction moge nun úber Des Ş2n. Th. und ưber Diefes Schreiben ifren Entid)luj nebmen. Dod wúnjdje id, Daß poldjer ganj zur Befriebigung Des Şrn. Th. ausfallen mòge, wogu id jebr gerne meine eigene Befriebigung, wenn id in biejer Sadje einige gu verlangen bătte, aufopfern moddte. E⿺ ift mir fatal, mit Månnern bon Ealenten in Eeinem guten Rerneh: men ju fehen, und es thut mir weh, in einem \&ande, wo id bisher mit po vieler unberdienten

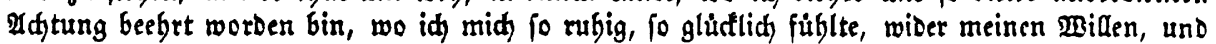
bel) Der beften $\mathfrak{A b f i d j t , ~ i n ~ e i n e ~} \mathfrak{A r t}_{\text {you }}$ Gehbe hineingegogen ju werben, Die meinem Serjen und meiner Denlungsart jo zuwieber ift, Das id berglid wúnidhe, fie moge, fo wie bie erfite, aud bie leg̨te meines ganjen lebens feinn.

Copenhagen, ben $20^{\mathfrak{n}}$ Már 1789.

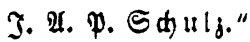

